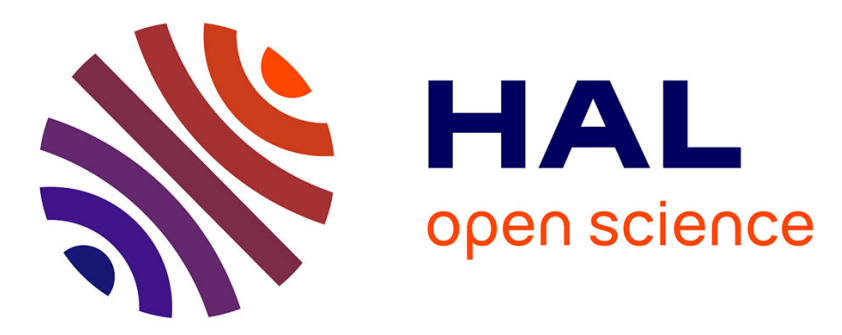

\title{
In- and out-of-plane longitudinal acoustic-wave velocities and elastic moduli in h-BN from Brillouin scattering measurements
}

\author{
Rafael J. Jimenez-Rioboo, Lluis Artus, R. Cusco, Takashi Taniguchi, \\ Guillaume Cassabois, Bernard Gil
}

\section{To cite this version:}

Rafael J. Jimenez-Rioboo, Lluis Artus, R. Cusco, Takashi Taniguchi, Guillaume Cassabois, et al.. In- and out-of-plane longitudinal acoustic-wave velocities and elastic moduli in h-BN from Brillouin scattering measurements. Applied Physics Letters, 2018, 112, pp.051905. 10.1063/1.5019629 . hal01697455

\section{HAL Id: hal-01697455 \\ https://hal.science/hal-01697455}

Submitted on 25 May 2021

HAL is a multi-disciplinary open access archive for the deposit and dissemination of scientific research documents, whether they are published or not. The documents may come from teaching and research institutions in France or abroad, or from public or private research centers.
L'archive ouverte pluridisciplinaire HAL, est destinée au dépôt et à la diffusion de documents scientifiques de niveau recherche, publiés ou non, émanant des établissements d'enseignement et de recherche français ou étrangers, des laboratoires publics ou privés. 


\section{In- and out-of-plane longitudinal acoustic-wave velocities and elastic moduli in h-BN from Brillouin scattering measurements}

R. J. Jiménez-Riobóo, L. Artús, R. Cuscó, T. Taniguchi, G. Cassabois, and B. Gil

Citation: Appl. Phys. Lett. 112, 051905 (2018);

View online: https://doi.org/10.1063/1.5019629

View Table of Contents: http://aip.scitation.org/toc/apl/112/5

Published by the American Institute of Physics

\section{Articles you may be interested in}

High efficiency and broadband acoustic diodes

Applied Physics Letters 112, 051902 (2018); 10.1063/1.5020698

SIMS study of oxygen diffusion in monoclinic $\mathrm{HfO}_{2}$

Applied Physics Letters 112, 051908 (2018); 10.1063/1.5020370

$2 \mathrm{kV}$ slanted tri-gate GaN-on-Si Schottky barrier diodes with ultra-low leakage current

Applied Physics Letters 112, 052101 (2018); 10.1063/1.5012866

Oxygen-assisted synthesis of hexagonal boron nitride films for graphene transistors Applied Physics Letters 111, 203103 (2017); 10.1063/1.5001790

Plate-type acoustic metamaterial with cavities coupled via an orifice for enhanced sound transmission loss Applied Physics Letters 112, 051903 (2018); 10.1063/1.5019602

Single photon extraction and propagation in photonic crystal waveguides incorporating site-controlled quantum dots

Applied Physics Letters 112, 051105 (2018); 10.1063/1.5007935

\section{Scilight}

Sharp, quick summaries illuminating the latest physics research

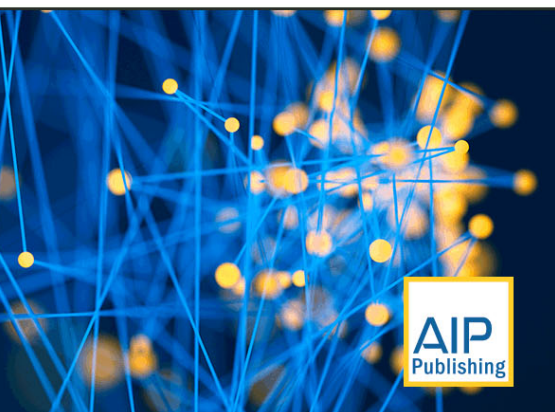




\title{
In- and out-of-plane longitudinal acoustic-wave velocities and elastic moduli in $\boldsymbol{h}$-BN from Brillouin scattering measurements
}

\author{
R. J. Jiménez-Riobóo, ${ }^{1}$ L. Artús, ${ }^{2}$ R. Cuscó, ${ }^{2}$ T. Taniguchi, ${ }^{3}$ G. Cassabois, ${ }^{4}$ and B. Gil ${ }^{4, a)}$ \\ ${ }^{1}$ Instituto de Ciencia de Materiales de Madrid (CSIC), Sor Juana Inés de la Cruz 3, E-28049 Madrid, Spain \\ ${ }^{2}$ Institut Jaume Almera (ICTJA-CSIC), Consejo Superior de Investigaciones Científicas, 08028 Barcelona, \\ Spain \\ ${ }^{3}$ National Institute for Materials Science, 1-1 Namiki, Tsukuba 305-0044, Japan \\ ${ }^{4}$ Laboratoire Charles Coulomb (L2C), UMR 5221 CNRS-Université de Montpellier, F-34095 Montpellier, \\ France
}

(Received 15 December 2017; accepted 12 January 2018; published online 30 January 2018)

\begin{abstract}
The elastic constants of high-quality, single-crystal hexagonal boron nitride ( $h$-BN) have been measured by means of high resolution Brillouin spectroscopy using a modified reflected light microscope. The sound propagation velocity in the $c$-axis direction and perpendicular to the $c$-axis have been obtained from the Brillouin frequency shift, with a proper account taken of the vast difference between the ordinary and extraordinary refractive indices recently reported in the highly anisotropic layered $h$-BN crystal. The elastic constants $c_{11}$ and $c_{33}$ obtained from the Brillouin experiments are somewhat lower than previous determinations based on inelastic x-ray measurements and confirm the overestimation of the $h$-BN elastic constants by ab initio calculations based on density functional perturbation theory in the local density approximation. Published by AIP Publishing.

https://doi.org/10.1063/1.5019629
\end{abstract}

Hexagonal boron nitride ( $h$ - $\mathrm{BN})$ is a layered compound that is emerging as a key material in the development of novel optoelectronic and electromechanical components in microand nanoscale devices. On the one hand, $h$-BN is a wide bandgap van der Waals crystal with a high thermal conductivity which displays an in-plane lattice constant similar to that of graphene. This makes it possible to form van der Waals stacked heterostructures and take advantage of the unique dielectric and thermal properties of $h$-BN for insulation and thermal management of 2D-material-based devices. ${ }^{1}$ On the other hand, $h$-BN nanotubes ${ }^{2}$ can be synthesized by a variety of methods, including chemical vapor deposition. Owing to their mechanical properties, high thermal conductivity and high-temperature oxidation resistance, they have great potential as electromechanical components in micro- and nanosystems, ${ }^{3}$ hardened structural composites, and energy-absorbing and radiation-protective composites for aircrafts. ${ }^{4}$

In electrically insulating materials, the low-temperature thermal conductivity is determined by the lattice dynamics of acoustic phonons, which is closely related to the elastic moduli. ${ }^{5}$ Despite the large technological interest of $h$-BN that is connected with its elastic properties, only a few experimental works on the elastic moduli can be found in the literature. The available data show a large scatter, most probably related to the poor quality of the pyrolytic ${ }^{5}$ or sputtered ${ }^{6}$ $h$-BN samples used in the previous studies. The thermal and elastic properties of pyrolytic boron nitride strongly depend on the sample morphology, namely, on the in-plane crystallite size and the point-defect distribution. ${ }^{5}$ Theoretical calculations based on anisotropic elasticity theory showed that the strain energy density for a biaxially strained $h$-BN single crystal is minimized when the basal plane is tilted roughly

\footnotetext{
${ }^{a)}$ On leave at the Ioffe Institute St. Petersburg, Polytekhnicheskaya 26, St. Petersburg 194021, Russia
}

half-way between the substrate surface and the plane normal. ${ }^{7}$ This differs from the experimental observation of basal $h$-BN planes being perpendicular to the film plane in turbostratic $\mathrm{BN}^{8}{ }^{8}$ and the primary role of plastic deformations in determining the film texture was suggested. ${ }^{7}$ More recent studies of $h$ BN thin films deposited by RF magnetron sputtering reported polycrystalline films with the $c$ axes of the crystallites randomly oriented parallel to the film plane and amorphous grain boundaries. ${ }^{6}$ The dispersion of surface acoustic waves in Brillouin scattering experiments on such thin films revealed an elastic anisotropy. The modulus for in-plane directions was stiffer than for the direction perpendicular to the film, but the anisotropy was significantly lower than that expected from a hexagonal layered structure. More recently, the elastic moduli of single-crystalline $h$-BN were experimentally determined from inelastic x-ray scattering measurements. ${ }^{9}$ These measurements revealed discrepancies with theoretical ab initio calculations of the elastic moduli, ${ }^{10}$ particularly regarding the in-plane stiffness ( $c_{11}$ elastic constant).

Here, we present high-resolution Brillouin scattering measurements performed on high-quality $h$-BN single-crystals that provide an experimental direct optical determination of the acoustic-wave velocities along the $c$-axis and perpendicular to the $c$-axis. High quality $h$-BN single crystals were synthesized at $4.5 \mathrm{GPa}$ and $1500^{\circ} \mathrm{C}$ using barium boron nitride as a solvent in the modified belt-type high-pressure and high-temperature apparatus. ${ }^{11}$ The samples were platelets of a few $\mathrm{mm}$ in the lateral dimensions and a thickness of a few hundred $\mu \mathrm{m}$. Because of the size and shape of the $h$-BN samples, high resolution Brillouin spectroscopy was performed with the aid of an inhouse modified reflected light Olympus BX51 microscope with a $\times 50$ SLMPlan Olympus objective. Brillouin spectra were recorded in the backscattering configuration using a Sandercock $3+3$ pass tandem Fabry-Pérot interferometer. ${ }^{12}$ The mechanical shutter of the Sandercock spectrometer was 
set to block the specular reflected light. The light source was a diode-pumped solid state laser operating at a wavelength of $\lambda_{0}=532 \mathrm{~nm}$. By using the microscope, Brillouin scattering measurements could be performed on the (0001) crystallographic plane and on a lateral facet perpendicular to it. Brillouin scattering measurements were carried out at several spots on three different $h$-BN samples, and within experimental error, the same results were consistently found.

In micro-Brillouin spectroscopy, it is only possible to use backscattering geometry. In the backscattering geometry, the selection rules favor the longitudinal mode, and unless significant light depolarization occurs, no coupling with the acoustic shear modes takes place. ${ }^{13}$ Brillouin scattering provides information about the acoustic phonon branches at a given wave vector. The acoustic wave vector for backscattering geometry is

$$
q_{b}=\frac{4 \pi n}{\lambda_{0}},
$$

where $n$ is the refractive index. The hypersonic sound propagation velocity can be calculated from the measured Brillouin frequency shift $f_{B}$ as

$$
v=\frac{2 \pi f_{B}}{q_{b}} .
$$

Thus, using Eq. (1), the hypersonic velocity can be written as

$$
v=\frac{f_{B} \lambda_{0}}{2 n}
$$

The elastic moduli $c_{11}$ and $c_{33}$ can then be determined from the propagation velocity of the longitudinal acoustic (LA) modes along the [100] and [001] directions, respectively, as $c_{11}=\rho v^{2}(\mathrm{LA}[100])$ and $c_{33}=\rho v^{2}(\mathrm{LA}[001])$. Here, $\rho=2.28$ $\mathrm{g} \mathrm{cm}^{-3}$ is the $h$-BN density as obtained from the lattice parameters determined by $\mathrm{x}$-ray diffraction. ${ }^{9,14}$

As can be seen from Eq. (3), in backscattering geometry, the hypersonic velocity depends heavily on the refractive index. Therefore, to extract reliable values of the sound velocity and the elastic moduli, a good knowledge of the refractive index is necessary, particularly in strongly anisotropic crystals, in which the ordinary and extraordinary refractive indices may substantially differ. Recent optical transmission measurements have revealed that $h$-BN displays a giant optical anisotropy, with the values of the refractive index of $n_{o}=2.304$ and $n_{e}=1.60$ for the ordinary and extraordinary rays, respectively, at $\lambda_{0}=532 \mathrm{~nm} .{ }^{15}$ The precise determination of the refractive index of $h$ - $\mathrm{BN}$ is fundamental to extract accurate values for the in- and out-of-plane sound velocities and elastic moduli.

Figure 1 shows the micro-Brillouin spectrum obtained from a flat-shaped platelet of $h$-BN corresponding to the (0001) crystallographic plane (inset). The red arrow indicates the polarization direction of the incident laser beam. In backscattering geometry, the micro-Brillouin spectrum corresponds to the LA mode along the $c$-axis direction, the commonly called ZA mode. ${ }^{16}$ Therefore, it yields information about the out-of-plane sound propagation velocity and the $c_{33}$ elastic constant. In this case, the refractive index

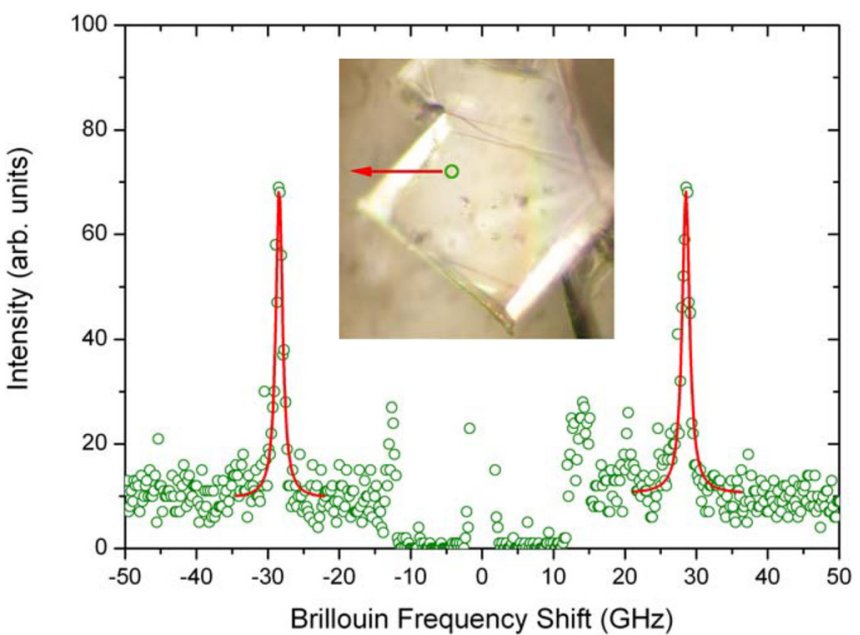

FIG. 1. High resolution Brillouin backscattering spectrum of the $h$-BN sample on a (0001) facet. In this configuration, the acoustic wave vector is parallel to the $c$-axis. Lorentzian line shapes (red lines) were fitted to the experimental data to accurately obtain the spectral frequency shift of the Brillouin peak. The inset shows the optical microscopy image of the (0001) platelet. The red arrow indicates the polarization direction of the incident light, and the green circle is the region probed by the Brillouin scattering measurements.

involved is the ordinary ray index, i.e., for light polarization contained in the (0001) plane. From the measured Brillouin frequency shift, using Eq. (3) and the ordinary refractive index $n_{o}=2.304$, the hypersound propagation velocity in the $c$-axis direction was determined to be $v_{\|}=3279 \pm 17 \mathrm{~m} \mathrm{~s}^{-1}$, and the corresponding elastic constant is $c_{33}=24.5 \pm 0.2 \mathrm{GPa}$.

Figure 2 shows the micro-Brillouin spectrum obtained on a lateral facet of the $h$-BN platelet. The contrast-enhanced image in the inset reveals the laminar morphology perpendicular to the $c$-axis in these facets. The micro-Brillouin spectrum was recorded with the incident laser polarization parallel to the $c$-axis, as depicted by the red arrow in the

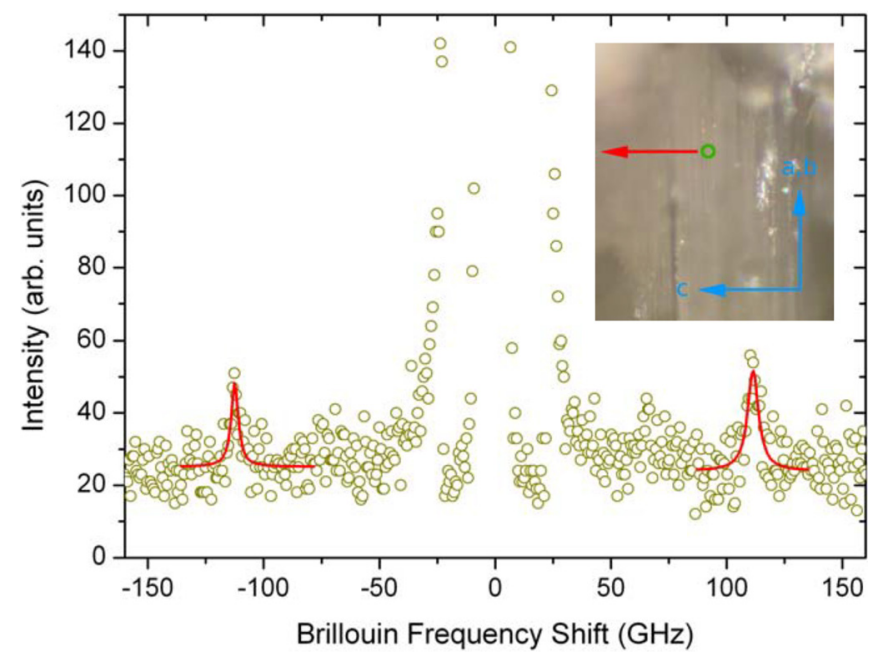

FIG. 2. Same as in Fig. 1 for a crystallographic plane perpendicular to (0001) (lateral facet). In this configuration, the acoustic wave vector lies within the $a, b$ crystallographic plane. The contrast-enhanced optical microscopy image shown in the inset reveals a laminar morphology perpendicular to the $c$-axis. In the inset, the region probed by the Brillouin scattering measurements and the polarization direction of the incident light are indicated by the green circle and the red arrow, respectively. The blue arrows indicate the crystallographic directions. 
TABLE I. In-plane and out-of-plane $c_{11}$ and $c_{33}$ elastic moduli of $h$-BN. Comparison of the values determined by Brillouin scattering with the experimental and theoretical values available in the literature.

\begin{tabular}{lcccc}
\hline \hline & References & $c_{11}(\mathrm{GPa})$ & $c_{33}(\mathrm{GPa})$ & Method \\
\hline Experiment & This work & 788 & 24.5 & Brillouin scattering: normal backscattering on $a$ and $c$ faces of a single crystal \\
& 9 & 811 & 27.0 & Inelastic x-ray scattering \\
Theory & 6 & 44 & 293 & Brillouin scattering: dispersion of the Rayleigh and first Sezawa mode. Polycrystalline thin \\
& 17 & 802.5 & 31.2 & film with $c$ axes mainly oriented parallel to the film plane \\
& 10 & 951.5 & 28.2 & Central force model \\
\hline \hline
\end{tabular}

inset, where the probed scattering volume is indicated by the green circle. In this geometry, the wavevector involved in Brillouin scattering [Eq. (1)] is determined by the extraordinary refractive index $n_{e}=1.60$. The relevant modes in this configuration are the in-plane LA modes, which are much stiffer than the interlayer ZA modes, and therefore, a much higher hypersound propagation velocity is expected. From the measured Brillouin frequency shift, Eq. (3) yields the hypersound propagation velocity perpendicular to the $c$-axis direction $v_{\perp}=18595.6 \pm 108 \mathrm{~m} \mathrm{~s}^{-1}$, and the corresponding elastic constant is $c_{11}=788.4 \pm 9 \mathrm{GPa}$.

A comparison between our Brillouin scattering results and the experimental and theoretical determinations of the $c_{11}$ and $c_{33}$ elastic constants found in the literature is given in Table I. The elastic moduli obtained from the Brillouin scattering experiments are in good agreement with the set of elastic moduli reported by Bosak et al., ${ }^{9}$ which were estimated in a self-consistent way from inelastic x-ray scattering data. The $c_{11}$ value we find is only $\sim 3 \%$ lower than the value obtained in Ref. 9. These $c_{11}$ values are in contrast with theoretical predictions of the elastic moduli based on density functional perturbation theory (DFT) in the local density approximation (LDA), which heavily overestimate the $c_{11}$ elastic constant by $\sim 20 \%$. The $c_{33}$ elastic constant is also overestimated by the theoretical calculations, and our Brillouin results are $\sim 10 \%$ lower than those of Bosak et al. ${ }^{9}$ This elastic constant, which is related to the weak interlayer bonding, is probably more sensitive to the sample preparation conditions, as it was found to vary significatively in pyrolytic samples with different interlayer spacings. ${ }^{5}$

The longitudinal-wave elastic constant was measured in polycrystalline cubic $(c$-BN) and wurtzite-type $\mathrm{BN}(w$-BN) by picosecond ultrasound spectroscopy. ${ }^{18}$ The elastic constants are similar in both phases, and they were found to be 945 and $930 \mathrm{GPa}$ for $c$-BN and $w$-BN, respectively. These values are in good agreement with DFT calculations in the LDA approximation. ${ }^{18}$ The in-plane elastic modulus measured in layered $h$-BN is sizably lower than that of $c$ - and $w$-BN. This is in contrast with ab initio calculations in the LDA approximation, which yield values closer to those found in $c$ - and $w$-BN. Furthermore, the elastic anisotropy of $h$ - $\mathrm{BN}$ is remarkably high, with an in-plane/out-of-plane elastic modulus ratio of $c_{11} / c_{33} \sim 32$. This is to be compared with much lower values reported in other layered materials in which the layers are also weakly bound by van der Waals interactions. For instance, the elastic modulus anisotropy ratio is 3.2 in $\mathrm{GaS}^{19}$ and 4.6 in $\mathrm{MoS}_{2}{ }^{20}$ This fact, together with the giant optical anisotropy recently reported, ${ }^{15}$ singles out $h$-BN as one of the most heavily anisotropic materials in the family of 2D-like van der Waals crystals which form the building blocks for novel atomically thin flexible electronics. A better understanding of the effect of the interlayer van der Waals interactions on the overall elastic properties of $h$-BN that goes beyond the local DFT description should be desirable. Hybrid functional calculations properly including van der Waals interactions and screening have been used in other layered compounds ${ }^{21}$ and could possibly provide a better account of the great anisotropy observed in the layered $h$-BN crystal.

In summary, we have measured the hypersound velocity and the elastic constants of $h$-BN in the $c$-axis direction and in the plane perpendicular to the $c$-axis by performing high resolution Brillouin backscattering experiments on a highquality single crystal. The direct optical determination of the $c_{11}$ and $c_{33}$ elastic constants, taking into account the giant optical anisotropy of $h$-BN, yields values slightly lower than previous determinations based on $\mathrm{x}$-ray inelastic scattering. These experimental results confirm that the elastic moduli of $h$-BN are clearly overestimated by existing local $a b$ initio calculations. Therefore, an improvement of the description of weak van der Waals interactions in $h$-BN appears to be necessary to achieve a better accord of the theory with the observed elastic properties.

This work was supported by the Spanish MINECO/ FEDER under Contract Nos. MAT2015-71035-R and MAT2015-65356-C3-1-R. B.G. acknowledges the Russian Megagrant program (Ioffe Institute of RAS, Contract No. 14.W03.31.0011). T.T. acknowledges the support from the Elemental Strategy Initiative conducted by the MEXT, Japan, and JSPS KAKENHI Grant No. JP15K21722. R.J.J.R. would like to thank J. K. Krüger from Universität des Saarlandes (Germany) for useful suggestions.

${ }^{1}$ T. Roy, M. Tosun, J. S. Kang, A. B. Sachid, S. B. Desai, M. Hettick, C. C. Hu, and A. Javey, ACS Nano 8, 6259 (2014).

${ }^{2}$ N. G. Chopra, R. J. Luyken, K. Cherrey, V. H. Crespi, M. L. Cohen, S. G. Louie, and A. Zettl, Science 269, 966 (1995).

${ }^{3}$ A. P. Suryavanshi, M.-F. Yu, J. Wen, C. Tang, and Y. Bando, Appl. Phys. Lett. 84, 2527 (2004).

${ }^{4}$ M. L. Cohen and A. Zettl, Phys. Today 63(11), 34 (2010).

${ }^{5}$ L. Duclaux, B. Nysten, J.-P. Issi, and A. W. Moore, Phys. Rev. B 46, 3362 (1992).

${ }^{6}$ T. Wittkowski, P. Cortina, J. Jorzick, K. Jung, and B. Hillebrands, Diamond Relat. Mater. 9, 1957 (2000).

${ }^{7}$ G. F. Cardinale, D. L. Medlin, P. B. Mirkarimi, K. F. McCarty, and D. G. Howitt, J. Vac. Sci. Technol., A 15, 196 (1997).

${ }^{8}$ D. L. Medlin, T. A. Friedmann, P. B. Mirkarimi, P. Rez, M. J. Mills, and K. F. McCarty, J. Appl. Phys. 76, 295 (1994).

${ }^{9}$ A. Bosak, J. Serrano, M. Krisch, K. Watanabe, T. Taniguchi, and H. Kanda, Phys. Rev. B 73, 041402(R) (2006). 
${ }^{10}$ N. Ohba, K. Miwa, N. Nagasako, and A. Fukumoto, Phys. Rev. B 63, 115207 (2001).

${ }^{11}$ T. Taniguchi and K. Watanabe, J. Cryst. Growth 303, 525 (2007).

${ }^{12}$ J. R. Sandercock, in Light Scattering in Solids III: Recent Results, edited by M. Cardona and G. Güntherodt (Springer Berlin Heidelberg, Berlin, Heidelberg, 1982), pp. 173-206.

${ }^{13}$ R. Vacher and L. Boyer, Phys. Rev. B 6, 639 (1972).

${ }^{14}$ W. Paszkowicz, J. Pelka, M. Knapp, T. Szyszko, and S. Podsiadlo, Appl. Phys. A 75, 431 (2002).

${ }^{15}$ A. Segura, L. Artús, R. Cuscó, T. Taniguchi, G. Cassabois, and B. Gil, "Natural optical anisotropy of $h$-BN: Highest giant birefringence in a bulk crystal through the mid-infrared to ultraviolet range," Phys. Rev. Mater. (in press).

${ }^{16}$ R. Cuscó, B. Gil, G. Cassabois, and L. Artús, Phys. Rev. B 94, 155435 (2016).

${ }^{17}$ J. F. Green, T. K. Bolland, and J. W. Bolland, J. Chem. Phys. 64, 656 (1976).

${ }^{18}$ A. Nagakubo, H. Ogi, H. Sumiya, K. Kusakabe, and M. Hirao, Appl. Phys. Lett. 102, 241909 (2013).

${ }^{19}$ A. Polian, M. Grimsditch, M. Fischer, and M. Gatulle, J. Phys. Lett. 43, 405 (1982).

${ }^{20}$ J. Feldman, J. Phys. Chem. Solids 37, 1141 (1976).

${ }^{21}$ H. Peelaers and C. G. Van de Walle, J. Phys. Chem. C 118, 12073 (2014). 